\title{
Brain Mechanisms Supporting Discrimination of Sensory Features of Pain: A New Model
}

\author{
Yoshitetsu Oshiro, ${ }^{1}$ Alexandre S. Quevedo, ${ }^{1}$ John G. McHaffie, ${ }^{1}$ Robert A. Kraft, ${ }^{2}$ and Robert C. Coghill ${ }^{1}$ \\ ${ }^{1}$ Department of Neurobiology and Anatomy, Wake Forest University School of Medicine, and ${ }^{2}$ Department of Biomedical Engineering, Division of \\ Radiologic Sciences, Wake Forest University School of Medicine, Winston-Salem, North Carolina 27157-1010
}

\begin{abstract}
Pain can be very intense or only mild, and can be well localized or diffuse. To date, little is known as to how such distinct sensory aspects of noxious stimuli are processed by the human brain. Using functional magnetic resonance imaging and a delayed match-to-sample task, we show that discrimination of pain intensity, a nonspatial aspect of pain, activates a ventrally directed pathway extending bilaterally from the insular cortex to the prefrontal cortex. This activation is distinct from the dorsally directed activation of the posterior parietal cortex and right dorsolateral prefrontal cortex that occurs during spatial discrimination of pain. Both intensity and spatial discrimination tasks activate highly similar aspects of the anterior cingulate cortex, suggesting that this structure contributes to common elements of the discrimination task such as the monitoring of sensory comparisons and response selection. Together, these results provide the foundation for a new model of pain in which bidirectional dorsal and ventral streams preferentially amplify and process distinct sensory features of noxious stimuli according to top-down task demands.
\end{abstract}

\section{Introduction}

In many sensory modalities, different sensory dimensions are processed by different brain networks. For example, distinct ventral "what" streams and dorsal "where" streams have been shown to be involved in the processing of visual, auditory, and innocuous somatosensory information (Goodale and Milner, 1992; Ungerleider and Haxby, 1994; Romanski et al., 1999; Rauschecker and Tian, 2000; Anourova et al., 2001; Reed et al., 2005; Barrett and Hall, 2006). The ventral stream plays a major role in the perceptual identification of objects, such as form, color, and features, while the dorsal stream mediates the coding of spatial characteristics and motion (Goodale and Milner, 1992; Ungerleider and Haxby, 1994; Romanski et al., 1999; Rauschecker and Tian, 2000; Anourova et al., 2001; Barrett and Hall, 2006). In the visual system, the ventral stream connects the striate area, ventral portions of the prestriate area (especially V4), inferior temporal cortical regions, and ventrolateral prefrontal cortex (Zeki, 1983; Desimone and Schein, 1987; Tanaka, 1997). The dorsal stream connects the striate area, dorsal parts of the prestriate area, superior parietal cortical regions, and dorsolateral prefrontal cortex (Sakata and Kusunoki, 1992; Movshon and Newsome, 1996; Colby and Goldberg, 1999).

Current conceptual models of pain do not incorporate such distinctions in the processing of spatial versus nonspatial aspects of nociceptive information (Melzack and Casey, 1968; Treede et

Received Nov. 18, 2008; revised Sept. 9, 2009; accepted Sept. 28, 2009.

This study was supported by National Institutes of Health Grant R01 NS39426. We thank FMRIB Image Analysis Group (Oxford University) for the FSL analysis software. We thank Christos Constantinidis for his helpful comments on this manuscript.

Correspondence should be addressed to Dr. Robert C. Coghill, Department of Neurobiology and Anatomy, Wake Forest University School of Medicine, Winston-Salem, NC 27157-1010. E-mail: rcoghill@wfubmc.edu. DOI:10.1523/JNEUROSCI.5538-08.2009

Copyright $\odot 2009$ Society for Neuroscience ～0270-6474/09/2914924-08\$15.00/0 al., 1999). Previously, using a combination of both psychophysical and functional magnetic resonance imaging (fMRI) techniques, we identified a set of brain areas involved in spatial discrimination of pain (Oshiro et al., 2007). Discrimination of spatial features of pain involves significant frontoparietal interactions much like the visual and auditory systems (Kulkarni et al., 2005; Oshiro et al., 2007; Porro et al., 2007). Most importantly, the posterior parietal cortex and dorsolateral prefrontal cortex activation during spatial discrimination of pain is consistent with the dorsal "where" stream of other modalities.

Since spatial aspects of pain are processed in the dorsal stream, how are nonspatial intensity-related aspects of pain processed? One possibility is that a ventrally directed processing stream analogous to that of the visual and auditory systems may be involved. A ventrally directed tactile processing pathway leads from primary somatosensory cortex (SI) to the frontal cortex and the temporal lobe limbic structures via relays in secondary somatosensory cortex (SII) and the insular cortex in monkeys (Mesulam and Mufson, 1982; Mufson and Mesulam, 1982; Friedman et al., 1986). To test the hypothesis that intensity discrimination of pain is processed preferentially by an architecture similar to the "what" stream of other sensory modalities, we examined brain activation during a delayed-match-to-sample intensity discrimination task. Intensity discrimination-related activation was then compared with previously published spatial discrimination findings to gain insights into distinctions in the processing of these sensory features of pain.

\section{Materials and Methods}

Subjects. The study was performed on 12 healthy volunteers, six females and six males (age 22-39 years, mean: 29 years). Nine subjects were right handed, and three were left handed. Subjects were white (two males, three females), Asian (two males), Hispanic (one male), and AfricanAmerican (one male, three females). All subjects gave written, informed 


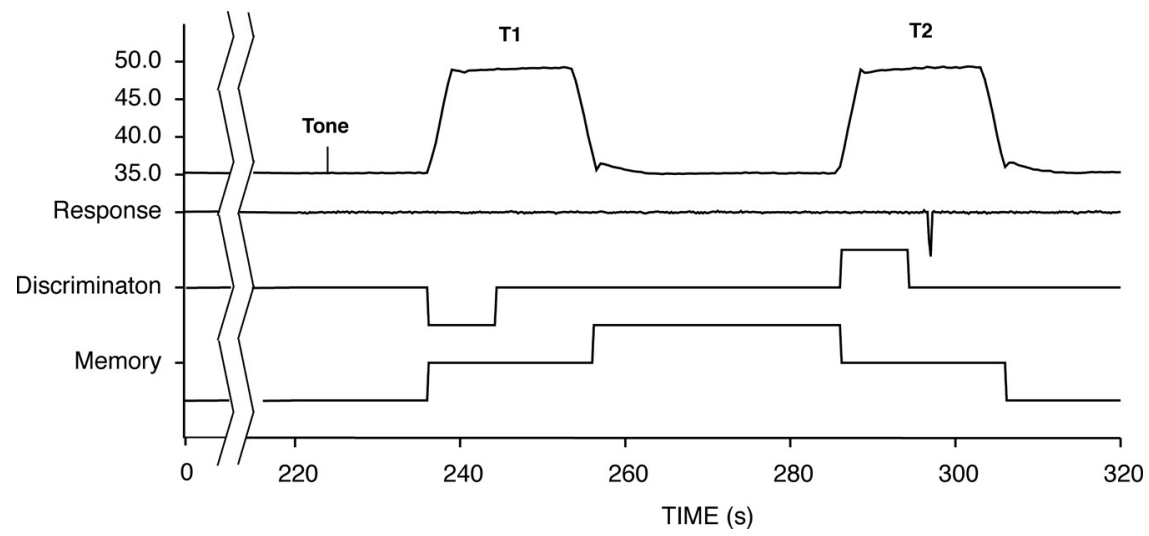

Figure 1. The temporal sequence of the experimental task. Pairs of noxious heat or innocuous cool stimuli were applied to the back of the lower leg. Before the termination of the second stimulus ( $\mathrm{T} 2: 48,48.5,49,50^{\circ} \mathrm{C}$ for noxious heat, $20,15^{\circ} \mathrm{C}$ for innocuous cool), subjects had to indicate that the intensity of $T 2$ was the same or was different from that of the first stimulus $\left(T 1: 48^{\circ} \mathrm{C}\right.$ for noxious heat, $20^{\circ} \mathrm{C}$ for innocuous cool). Discrimination-related brain activity was identified using regressors determined by the duration of the evaluation (i.e., response latency) of pairs of equally intense stimuli $\left(48^{\circ} \mathrm{C}-48^{\circ} \mathrm{C}\right.$ or $20^{\circ} \mathrm{C}-20^{\circ} \mathrm{C}$ ). Additional regressors were used to identify brain activation that was related to memory and to perceived pain (or cool).

consent acknowledging the following: (1) that they would experience painful stimuli, (2) that all methods and procedures were clearly explained, and (3) that they were free to withdraw from the experiment at any time without prejudice. All procedures were approved by the Institutional Review Board of Wake Forest University School of Medicine.

Psychophysical training. All subjects were initially trained with thirtytwo 5 -s-duration stimuli $\left(35-49^{\circ} \mathrm{C}\right)$ to give them experience rating pain. They then received the exact stimulus paradigms that were subsequently used during the fMRI session to ensure that they could adequately perform the discrimination task. Additionally, this procedure served to familiarize them with the temporal sequence of stimuli within a series to minimize variations in cognitive components such as expectation and anxiety.

Stimulation procedures. A thermal stimulator with a $16 \times 16 \mathrm{~mm}$ contact surface (Medoc TSA II) was used for noxious heat and innocuous cool stimulation. The device was attached with a Velcro strap to the posterior aspect of the left calf. A baseline temperature of $35^{\circ} \mathrm{C}$ was maintained. The stimulus temperature was changed with rise and fall rates of $6^{\circ} \mathrm{C} / \mathrm{s}$. To minimize sensitization or adaptation, each experimental series (see below) was performed on previously unstimulated skin sites.

Experimental task. A two-alternative forced-choice paradigm using pairs of thermal stimuli was used to identify brain regions involved in the discrimination of pain intensity (Fig. 1). This task and subsequent analyses were designed to parallel our previous study of spatial discrimination of pain as closely as possible (Oshiro et al., 2007). For each discrimination trial, $25 \mathrm{~s}$ after the initiation of the experimental task, a $1 \mathrm{~s}$ tone was delivered to the subject. After a $5 \mathrm{~s}$ period, a noxious heat stimulus $\left(48^{\circ} \mathrm{C}\right.$ ) was applied for $20 \mathrm{~s}$ (T1). A 30 s memory period followed the heat stimulation, after which a second $20 \mathrm{~s}$ stimulus was delivered at varied temperatures $\left(\mathrm{T} 2: 48,48.5,49,50^{\circ} \mathrm{C}\right)$. Subjects were required to determine whether the $\mathrm{T} 2$ temperature was the same as or different from T1 by pushing a switch with the right index finger for "same" or the right middle finger for "different." Subjects were instructed that this decision was to be made before the termination of T2. Each 320 s series was limited to three pairs of stimuli to minimize fatigue, habituation, and/or sensitization. Thus, during each series, each subject performed three intensity discriminations. All subjects underwent eight such series of noxious heat stimuli. An identical intensity discrimination task using pairs of innocuous cool stimuli $\left(\mathrm{T} 1: 20^{\circ} \mathrm{C} ; \mathrm{T} 2: 20,15^{\circ} \mathrm{C}\right)$ was used to determine whether similar brain networks were involved in discriminating the intensity of nonpainful stimuli. Cool stimuli were used instead of innocuous warm stimuli because noxious heat stimuli can produce a pronounced suppression of activity in warmth afferents and warmth sensation (LaMotte and Campbell, 1978; Darian-Smith et al., 1979). Subjects underwent four series of cool discrimination. Stimulus temperatures were pseudorandomized, and task order was counterbalanced across subjects.
Psychophysical assessment and analysis. For both the training and fMRI acquisition series, subjects' discrimination responses were recorded using a digital chart recorder (PowerLab: ADInstruments). These real-time data then were processed using customized programs within the IDL software package (Research Systems, CO). Response latencies and error rates were examined using repeatedmeasures ANOVAs to identify effects of stimulus temperature on the ability to discriminate intensity. These data were additionally used to construct regressors for the fMRI analysis (see below).

Subjective evaluations of task difficulty, pain intensity, and pain unpleasantness were acquired at the end of each series with a visual analog scale (VAS). The scales had a $0-10$ range, were $15 \mathrm{~cm}$ long, and were anchored with the words "Not at all Difficult"-"Most Difficult imaginable," "No Pain Sensation""Most Intense Pain Imaginable," "Not at all Unpleasant"-"Most Unpleasant Imaginable," respectively. Subjects were also queried as to the mnemonic strategy they used during the memory interval. Subjects were instructed that these ratings should reflect their overall experience of the whole series. Identical postseries ratings were obtained in the previous spatial discrimination study (Oshiro et al., 2007).

Image acquisition and image processing. Functional data were acquired on a 1.5 T General Electric echo-speed Horizon LX scanner with a birdcage head coil (GE Medical Systems). For functional imaging, blood oxygenation level-dependent images were acquired continuously in each contiguous plane by using echo-planar imaging [echo time (TE), $40 \mathrm{~ms}$; repetition time (TR), $2 \mathrm{~s} ; 28 \times 5$-mm-thick slices; $3.72 \times 3.75 \mathrm{~mm}$ in-plane resolution; flip angle, $90^{\circ}$; no slice gap]. During fMRI acquisition series, subjects were requested to close their eyes. Tones cueing the initiation of the discrimination trials were delivered through headphones.

High-resolution structural scans were acquired using a threedimensional (3-D) spoiled gradient-echo (3D Inversion SPGR) sequence (inversion time, $600 \mathrm{~ms}$; TR, $9.1 \mathrm{~ms}$; flip angle, 20 TE, $1.98 \mathrm{~ms}$; matrix, $256 \times 196$; section thickness, $1.5 \mathrm{~mm}$ with no gap between sections; number of sections, 124; in-plane resolution, $0.9375 \times 0.9375 \mathrm{~mm}$; field of view, $24 \mathrm{~cm}$ ).

The functional image analysis package FSL [Center for Functional Magnetic Resonance Imaging of the Brain (FMRIB), University of Oxford, Oxford, UK] was used for imaging processing and statistical analysis. The functional data were movement corrected, spatially smoothed by $5 \mathrm{~mm}$ with a 3-D isotropic Gaussian kernel, and temporally filtered by a nonlinear high-pass filter with a cutoff period of $75 \mathrm{~s}$. Each functional image was scaled by its mean global intensity (intensity normalization). Each subject's functional images were registered to their structural data using a seven-parameter linear 3-D transformation and then transformed into standard stereotaxic space (Talairach and Tournoux, 1988) (as defined by Montreal Neurologic Institute) using a 12-parameter linear 3-D transformation.

Statistical analysis of regional signal changes within the brain. We analyzed regional signal changes induced during memory, discrimination, noxious heat, and innocuous cool stimulation. Statistical analysis of these regional signal changes was performed on each acquisition series (first level analyses) using a general linear modeling approach with nonparametric local autocorrelation correction (Friston et al., 1995; Woolrich et al., 2001). In all analyses, the relationship of the predictive model function to MRI signal intensity was evaluated by calculating a $t$-statistic on a voxel-by-voxel basis. These $t$ values were then converted to $\mathrm{Z}$ scores to allow $p$ values to be calculated on the basis of Gaussian random field theory (Worsley et al., 1992; Friston et al., 1994; Forman et 
al., 1995). The predictive model functions for the general linear modeling analysis were derived as follows.

For analyses of regional signal changes induced by noxious heat or innocuous cool, stimulation periods were scaled as +1 , memory periods were scaled as 0 , and all rest periods were scaled as -1 (Fig. 1).

To examine brain mechanisms activated during the discrimination task, the real-time data from the chart recorder were processed using customized programs within the IDL software package. For analysis of discrimination, we selected only stimulus pairs in which $\mathrm{T} 2=\mathrm{T} 1$ to avoid confounds arising from the use of different temperatures. The first $75 \%$ of discrimination period (T2 onset-response choice) was selected for analysis to ensure that motor activity resulting from response selection did not confound assessment of discrimination activation. This period was scaled as +1 . For control periods, the corresponding time periods in $\mathrm{T} 1$ were scaled as -1 . To orthogonalize these analytic periods from the rest of the trial, other portions of the trial were scaled as 0 (Fig. 1). Since subjects were required to provide postseries ratings of pain, the cognitive activity associated with the formulation of these ratings would be a potential confound that would be present during both stimulation periods. However, since discrimination-related activation was identified by comparing the discrimination period of T2 with the corresponding period of T1, confounding effects of activity related to the postseries rating of pain would be minimized.

For analyses of regional signal changes induced by memory, the memory period between T1 and T2 was scaled as +1 and rest periods were scaled as -1 . Periods of thermal stimulation were scaled as 0 (Fig. 1). As above, since subjects were required to provide postseries ratings of pain, the cognitive activity associated with the memory of these ratings would be a potential confound that would be present during both rest and memory periods. However, since memory-related activation was identified by comparing the memory periods between $\mathrm{T} 1$ and $\mathrm{T} 2$ with the intertrial rest periods, confounding activity related to the postseries rating of pain would be minimized.

All regressors were convolved with a gammavariate model of the hemodynamic response (delay $6 \mathrm{~s}, \mathrm{SD} 3 \mathrm{~s}$ ) and its temporal derivative (Cohen, 1997; Josephs et al., 1997; Woolrich et al., 2001). They then were filtered temporally using the same parameters that were applied to the functional images.

We performed interseries fixed effects (second level) analyses within each subject separately for thermal stimulation, discrimination and memory, and proceeded to intersubject group analysis using a random effects model. These analyses were separately executed to facilitate the identification of brain regions that might participate in multiple aspects of the discrimination task (i.e., sensory processing, memory, and/or discrimination) and to parallel our previous study of spatial discrimination of pain (Oshiro et al., 2007). Clusters of voxels exceeding a $\mathrm{Z}$ score $>2.3$ and $p<0.05$ (mixed effect, corrected for multiple comparisons) were considered statistically significant (Worsley et al., 1992).
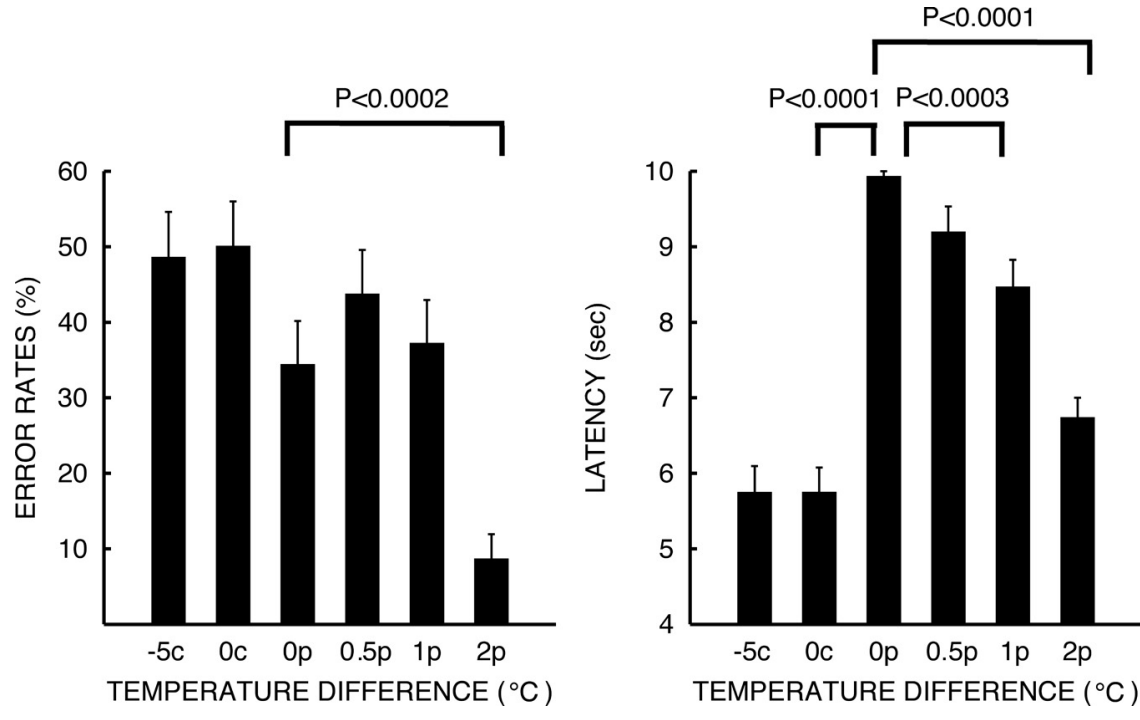

Figure 2. Behavioral responses during intensity discrimination (mean $\pm \mathrm{SEM}$ ). Discrimination of noxious heat pairs became progressively more difficult as temperature differences $\left(0^{\circ} \mathrm{C}: 0 \mathrm{p}, 0.5^{\circ} \mathrm{C}: 0.5 \mathrm{p}, 1^{\circ} \mathrm{C}: 1 \mathrm{p}, 2^{\circ} \mathrm{C}: 2 \mathrm{p}\right)$ between $\mathrm{T} 1$ and $\mathrm{T} 2$ stimuli decreased. Response latencies and error rates increased as stimuli were delivered with smaller temperature differences. Discrimination of pairs of innocuous cool stimuli $\left(0^{\circ} \mathrm{C}: 0 \mathrm{c},-5^{\circ} \mathrm{C}:-5 \mathrm{c}\right)$ was accomplished with latencies that were significantly shorter than those of comparable differences in noxious heat stimuli ( 0 c vs 0 p), but with error rates that were statistically indistinguishable. These latency differences may be due to the rapid adaptation that occurs during innocuous cool stimulation.

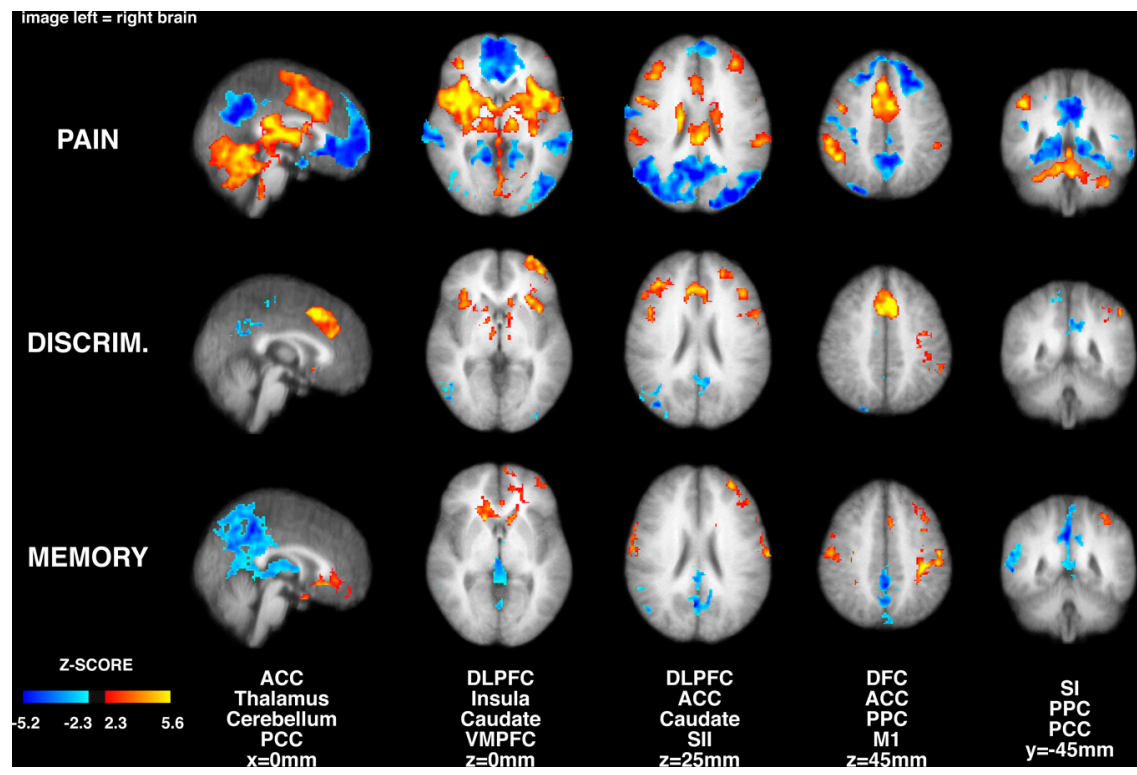

Figure 3. Brain activation and deactivation during painful heat stimulation, intensity discrimination, and intensity memory (image left $=$ right brain). Painful stimulation produced significant activation of numerous brain regions including the thalamus, SII, ACC, insular cortex, and prefrontal cortex. The anterior insula, prefrontal cortex, ACC, thalamus, and caudate nucleus were differentially activated during pain intensity discrimination, while SI was deactivated. During pain memory, the VMPFC was activated, in contrast to the deactivation seen in the pain versus rest comparisons. In all comparisons the PCC exhibited less activation than during rest. MI, Primary motor cortex. Slice locations are relative to standard stereotaxic space.

Comparisons of intensity discrimination-related versus spatial discriminationrelated activation. To better understand distinctions between processes subserving intensity discrimination versus spatial discrimination, previously published data from a parallel spatial discrimination study were qualitatively and quantitatively compared with brain activation data obtained during intensity discrimination (Oshiro et al., 2007). Regions of overlapping activation and unique activation were identified by combining binarized masks of $z$-score data from each study. These regions were then displayed on the averaged structural brain image from the two studies to provide a qualitative assessment of differences (and similari- 
ties) in activity produced during intensity versus spatial discrimination. Next, a random-effects analysis was performed to quantitatively determine whether regions uniquely activated during either task exhibited differential magnitudes of activity during intensity versus spatial discrimination.

The present investigation was designed from the beginning to parallel the spatial discrimination study as closely as possible- $-\mathrm{T} 1$ and $\mathrm{T} 2$ durations and interstimulus intervals were identical, response choices were identical (same/different choice with button presses from fingers on the right hand), task onset tones were identical, and postseries rating tasks were identical. These tasks were executed in separate sets of subjects in separate experiments to minimize confounds arising from covert attention to incorrect sensory dimensions that could occur in experiments where subjects alternated between spatial and intensity discrimination tasks. Thus, from the training session to the fMRI session, subjects in the spatial discrimination experiment only performed the spatial task, while subjects in the present investigation only performed the intensity discrimination task.

\section{Results}

\section{Psychophysics}

During discrimination of pairs of noxious heat stimuli (pain intensity VAS rating $=3.23 \pm 0.186$, mean \pm SEM), error rates and response latencies decreased significantly as the temperature difference increased (response latency: overall $F=51.47, p<$ $0.0001 ; 0^{\circ} \mathrm{C}$ vs $1^{\circ} \mathrm{C}$ difference: $F=27.89, p<0.0003 ; 0^{\circ} \mathrm{C}$ vs $2^{\circ} \mathrm{C}$ difference: $F=90.18, p<0.0001$; error rates: overall $F=5.79$, $p<0.0002 ; 0^{\circ} \mathrm{C}$ vs $2^{\circ} \mathrm{C}$ difference: $F=43.62, p<0.0001$ ) (Fig. 2 ). Response latencies and error rates were not significantly affected by perceptually small temperature differences $\left(5^{\circ} \mathrm{C}\right)$ during discrimination of innocuous cool stimuli (mean cool intensity VAS rating $=1.43 \pm 0.151$, mean \pm SEM). However, ANOVA detected significant differences in response latencies during noxious heat versus innocuous cool discrimination $\left(\operatorname{cool} 0^{\circ} \mathrm{C}\right.$ difference vs noxious heat $0^{\circ} \mathrm{C}$ difference: $F=270.49, p<$ 0.0001 ) (Fig. 2). These findings confirm that subjects were correctly performing the discrimination task.

\section{Pain- and cool-induced activation}

Group analysis of brain images revealed significant positive painrelated signal changes within the bilateral cerebellum, thalamus, globus pallidus/putamen (GP/PT), anterior insula, posterior insula/SII, anterior cingulate cortex (ACC), supplementary motor area (SMA), dorsolateral prefrontal cortex (DLPFC), dorsal frontal cortex (DFC), and posterior parietal cortex (PPC) (Fig. 3). Significant negative pain-related activation was detected in the bilateral posterior cingulate cortex (PCC), temporal cortex, DFC, and ventromedial prefrontal cortex (VMPFC). For innocuous cool stimulation, significant signal changes were evident within brain regions that were similar to those activated during pain (supplemental Fig. 1, available at www.jneurosci.org as supplemental material). These regions include the GP/PT, frontoparietal operculum, anterior insula, posterior insula/SII, DLPFC, DFC, ACC, PPC, PCC, and SMA. These results are consistent with previous studies (Coghill et al., 1999) and indicate that the subjects were sufficiently sensitive and that the stimuli were sufficiently robust to reliably detect activation of structures associated with the processing of information arising from thermal stimulation.

\section{Intensity discrimination-related activation}

Significant intensity discrimination-related activation was detected within portions of the bilateral anterior and medial thalamus, head of caudate, anterior insula, DLPFC, and ACC while
Table 1. Intensity discrimination-related and intensity memory-related activations and deactivations for pain

\begin{tabular}{|c|c|c|}
\hline Region & Discrimination & Memory \\
\hline \multicolumn{3}{|l|}{ Activation } \\
\hline \multicolumn{3}{|l|}{ VMPFC } \\
\hline Right & $(-)$ & $3.57(7522,24,44,-16)$ \\
\hline Left & $(-)$ & $4.03(7522,-14,38,-16)$ \\
\hline \multicolumn{3}{|l|}{ Thalamus } \\
\hline Right & $3.18(853,10,-12,-2)$ & $(-)$ \\
\hline Left & $3.19(853,-10,-12,4)$ & $(-)$ \\
\hline Caudate n. & (dorsal) & (ventral) \\
\hline Right & $3.75(853,10,12,8)$ & $4.12(7522,20,22,-2)$ \\
\hline Left & $4.3(504,-16,16,8)$ & $3.43(7522,-12,20,-2)$ \\
\hline \multicolumn{3}{|l|}{ Anterior insula } \\
\hline Right & $4.3(853,32,22,4)$ & $(-)$ \\
\hline Left & $4.93(1184,-32,18,-4)$ & $(-)$ \\
\hline \multicolumn{3}{|l|}{ Frontal pole (BA10) } \\
\hline Right & $(-)$ & $(-)$ \\
\hline Left & $4.35(1184,-34,62,2)$ & $4.43(7522,-32,48,18)$ \\
\hline \multicolumn{3}{|l|}{ DLPFC (BA9) } \\
\hline Right & $4.9(589,40,36,26)$ & $(-)$ \\
\hline Left & $4.98(791,-40,26,28)$ & $3.51(7522,-46,28,28)$ \\
\hline \multicolumn{3}{|l|}{$\mathrm{ACC}$} \\
\hline Right & $4.62(2270,6,12,42)$ & $(-)$ \\
\hline Left & $4.88(2270,-8,12,46)$ & $3.35(580,-4,18,42)$ \\
\hline Left M1/SI/PMA & $4.33(782,-52,-38,50)$ & $4.69(7522,-56,-16,40)$ \\
\hline \multicolumn{3}{|l|}{ PPC-IPL } \\
\hline Right & $(-)$ & $(-)$ \\
\hline Left & $3.12(782,-38,-50,40)$ & $4.83(7522,-44,-28,54)$ \\
\hline \multicolumn{3}{|l|}{ PPC-SPL } \\
\hline Right & $(-)$ & $(-)$ \\
\hline Left & $(-)$ & $(-)$ \\
\hline \multicolumn{3}{|l|}{ DFC (BA8) } \\
\hline Right & $(-)$ & $3.59(1335,56,-14,36)$ \\
\hline Left & $3.60(-32,-12,52)$ & $3.88(580,-20,6,58)$ \\
\hline Right SI (leg) & $(-)$ & $3.26(1335,10,-40,72)$ \\
\hline SII & $(-)$ & $(-)$ \\
\hline \multicolumn{3}{|l|}{ Deactivation } \\
\hline Right SI & $-3.28(530,10,-44,68)$ & $(-)$ \\
\hline LeftSI & $(-)$ & $-3.08(5056,-4,-44,70)$ \\
\hline Right BA19 & $-4.73(1396,52,-70,-12)$ & $(-)$ \\
\hline Left PCC & $-4.18(583,-8,-64,14)$ & $-4.5(5056,-6,-64,30)$ \\
\hline Right thalamus & $(-)$ & $-4.23(5056,18,-26,12)$ \\
\hline Left thalamus & $(-)$ & $-4.04(5056,-18,-22,12)$ \\
\hline
\end{tabular}

Peak Z-scores were obtained from group analysis. Cluster sizes and peak locations within significant clusters are listed within the parentheses as number of voxels and $x, y, z$ coordinates according to standard stereotaxic space. Note that a single statistically significant cluster can extend across multiple anatomic regions and have multiple local maxima. Caudate n., Caudate nucleus.

subjects were actively comparing the present intensity of a painful stimulus with that of a previous painful stimulus (Fig. 3, Table 1). Unilateral activation was detected in left PPC, left premotor area (PMA), left primary sensory and motor cortex (SI and M1), and left frontal polar cortex. Neither the leg region of SI nor SII was differentially activated during intensity discrimination. However, the right SI leg representation along with the occipital cortex (BA19) and left PCC exhibited significant deactivation during discrimination. During discrimination of innocuous cool intensity, bilateral portions of the medial thalamus, ACC and right posterior thalamus, left PMA and M1, and left periaqueductal gray (PAG) were activated (supplemental Fig. 1, available at www.jneurosci.org as supplemental material). Differential activation of SI (leg representation) and SII was not detected, as was the case with discrimination of noxious heat intensity.

At a qualitative level, brain activation during pain intensity discrimination was partially distinct from that previously ob- 
served during spatial discrimination of pain (Fig. 4). Spatial discrimination produced pronounced unique activation of regions such as the posterior parietal cortex and the right dorsolateral prefrontal cortex, while pain intensity discrimination produced unique activation of bilateral portions of the anterior insular cortex, more ventral portions of the prefrontal cortex, as well as the left caudate nucleus. Both discrimination tasks activated partially overlapping regions of the anterior cingulate cortex and the right caudate nucleus. Quantitative comparisons support the qualitative observations (Fig. 4). Portions of the right insular cortex $(z$ score $=3.76, x=34 \mathrm{~mm}, y=34$ $\mathrm{mm}, z=0 \mathrm{~mm})$, right putamen $(z$ score $=$ $3.45, x=24 \mathrm{~mm}, y=14 \mathrm{~mm}, z=0 \mathrm{~mm})$, left superior frontal gyrus $(z$ score $=3.98$, $x=-18 \mathrm{~mm}, y=20 \mathrm{~mm}, z=56 \mathrm{~mm})$,

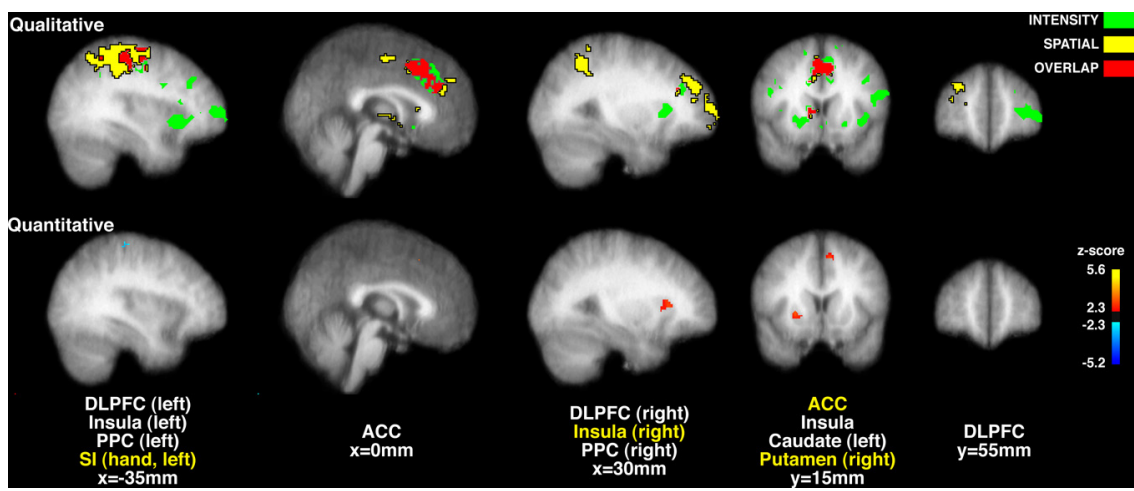

Figure 4. Qualitative (top row) and quantitative (bottom row) comparisons of brain activation during intensity and spatial discrimination of pain. Intensity discrimination (green) activated bilateral portions of the insular cortex, DLPFC, caudate nucleus and thalamus, and left PPC. Spatial discrimination (yellow) activated bilateral portions of the PPC, and right lateralized portions of the thalamus, DLPFC, and caudate nucleus. Both discrimination tasks activated overlapping regions (red) of the ACC, right caudate, and right DLPFC. At a quantitative level (yellow labels), intensity discrimination produced significantly greater activation than spatial discrimination (red/yellow) in portions of the right anterior insular cortex, right putamen, and left ACC. Spatial discrimination produced significantly greater activation of the left SI (hand area) (blue). Slice locations are relative to standard stereotaxic space.

and left ACC $(z$ score $=3.52, x=-10$

$\mathrm{mm}, y=16 \mathrm{~mm}, z=42 \mathrm{~mm}$ ) exhibited significantly greater activity during intensity discrimination than during spatial discrimination. Conversely, portions of the left primary somatosensory cortex (hand area, $z$ score $=3.63, x=-30 \mathrm{~mm}, y=-28 \mathrm{~mm}, z=58 \mathrm{~mm}$ ) exhibited significantly greater activity during spatial discrimination than during intensity discrimination.

\section{Intensity memory-related activation}

Subjects most frequently reported that they attempted to remember intensity itself while performing the intensity discrimination task. Subjects less frequently focused on other dimensions of the percept but never used verbal categorical strategies (Fig. 5). During the memory period between each pair of noxious heat stimuli, significant brain activation was detected within bilateral portions of the VMPFC, head of caudate, PMA, SI, and M1 (hand), and DFC. Unilateral activation was detected in the left DLPFC, left ACC, left PPC, and left frontal pole (Fig. 3). Significant deactivation was detected in bilateral thalamus, portions of the body of caudate, precuneus, regions of the left PCC, and SI (leg representation).

Memory-related activation that occurred between pairs of noxious stimuli overlapped partially with pain intensity discriminationrelated activation. The left PPC, PMA, SI, and M1 all exhibited substantial activation during both memory and discrimination (Fig. 5). More restricted regions in left head of caudate, DLPFC, and ACC also were activated during both tasks. Overlapping activation was detected in the hand regions of left M1, as well as related premotor and sensory cortices. Since subjects had to push the switches with fingers on their right hand to indicate their discrimination choice, this overlapping left motor activation likely represents motor planning. Importantly, no movements were required during the memory period, and discrimination-related brain activity was examined before subjects' responses. In contrast, memoryrelated and discrimination-related activation did not overlap in the right hemisphere. Memory-related activation for innocuous cool stimulation was found in bilateral ACC, bilateral head of caudate, right putamen, right anterior insula, left PPC, and left SI (supplemental Fig. 1, available at www.jneurosci.org as supplemental material).
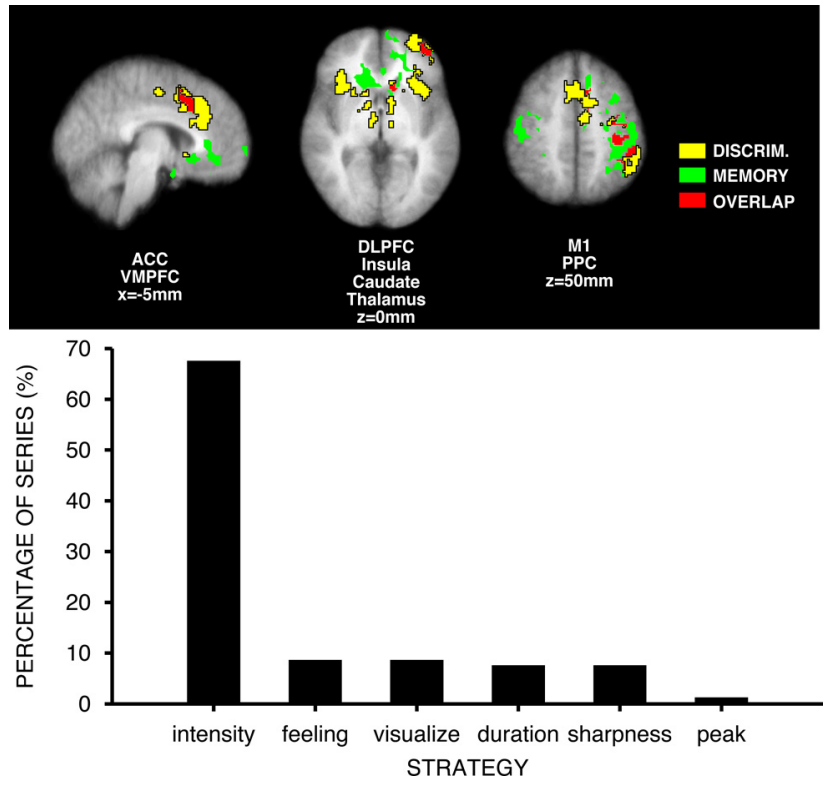

Figure 5. Brain activation during memory and discrimination of pain intensity. Memoryrelated activation overlapped partially with discrimination-related activation in left hemisphere, but not the right hemisphere. During interstimulus interval, subjects frequently ( $\sim 70 \%$ of trials) reported that they remembered the intensity of the $\mathrm{T} 1$ stimulus itself. Subjects rarely used verbal categorical strategies for the mnemonic phase of the intensity discrimination task. M1, Primary motor cortex. Slice locations are relative to standard stereotaxic space.

\section{Discussion}

Traditional models of brain mechanisms supporting pain emphasize the roles of the primary and secondary somatosensory cortices in the processing of sensory features of noxious stimuli (Melzack and Casey, 1968; Treede et al., 1999). Yet, these models make few distinctions about differential processing of different sensory dimensions and address the involvement of association cortices in sensory processing in a very limited manner. However, in other sensory modalities, substantial evidence indicates that spatial features of stimuli are processed by a dorsal stream involving the posterior parietal cortex and dorsolateral prefrontal cortex, whereas nonspatial features are processed by a ventrally 


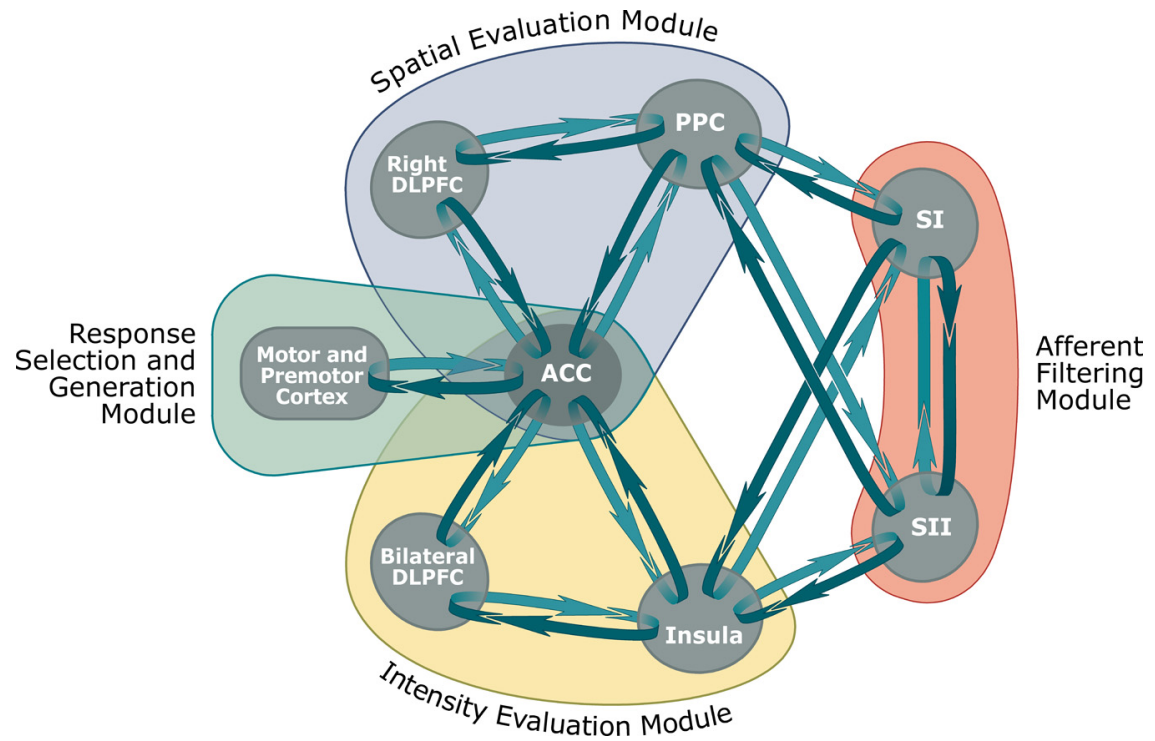

Figure 6. Cerebral cortical mechanisms supporting discrimination of sensory features of pain. The present data indicate that distinct modules are differentially engaged during discrimination of sensory features of pain. The somatosensory cortices (orange) process incoming afferent noxious information from the thalamus and are substantially modulated by top-down signals. This modulation involves the tuning of neural responses for optimal task performance-allowing appropriate information to be fed forward while minimizing the processing of irrelevant dimensions. Once the task-appropriate information has been initially filtered, it is then transmitted to higher cortical loops. Intensity-related information is preferentially processed by a ventrally located module (yellow) involving the insular cortex, bilateral portions of the prefrontal cortex, and cingulate cortex. Spatial information is preferentially processed by a dorsally located module (blue) that involves the PPC, right DLPFC, and ACC. The architecture of each of these modules is sufficient to support a cyclic comparison of information from previously experienced stimuli with incoming afferent information. Both intensity and spatial discrimination tasks activate overlapping portions of the ACC, indicating that this brain region participates in a process common to both tasks, such as response selection. The ACC is closely connected with SMA, premotor, and primary motor cortical regions, and together, these regions form a module that generates and communicates a final decision about the nature of the sensory features of the discriminated stimuli (green).

In contrast to the ventrally directed processing stream activated during pain intensity discrimination, spatial information can be transferred via a dorsally directed cortical route from SI and SII to the PPC and then to the DLPFC (Selemon and Goldman-Rakic, 1985, 1988; Neal et al., 1987). Consistent with this anatomic architecture, spatial discrimination of pain activated bilateral portions of the posterior parietal cortex and right lateralized portions of the prefrontal cortex (Figs. 4, 6) (Oshiro et al., 2007).

Direct quantitative comparisons confirmed that portions of the ventrally directed processing stream were significantly more active during intensity versus spatial discrimination. Such regions included the right anterior insular cortex. Although spatial discrimination was examined in a separate study in different subjects, the present study of intensity discrimination was designed in parallel with the spatial study to ensure that both paradigms were as comparable as possible. Moreover, by examining spatial and intensity discrimination in separate studies, confounds due to subject's covert attention to the incorrect sensory dimension were substantially minimized.

The direction of attention frequently involves an enhancement of neural activity relevant to the target and a simul-

directed stream associated with structures such as the temporal lobe and ventral portions of the prefrontal cortex. Discrimination of pain intensity, a nonspatial sensory feature, activates a network of brain regions encompassing the anterior insular cortex and the prefrontal cortex. This ventrally directed processing stream is partially distinct from that activated during the discrimination of spatial features of noxious stimuli and, thus, provides evidence that different sensory features of noxious stimuli are preferentially processed by distinct neural systems (Figs. 4, 6) (Oshiro et al., 2007).

Pain intensity discrimination uniquely activated bilateral portions of the anterior insular cortex and prefrontal cortex, and produced a predominately unilateral activation of the left prefrontal cortex in the vicinity of the frontal pole. This pattern of activation is largely consistent with anatomical evidence of a somatosensory processing pathway that leads from SI to SII to the prefrontal cortex via connections in the insular cortex (Mesulam and Mufson, 1982; Mufson and Mesulam, 1982; Friedman et al., 1986; Felleman and Van Essen, 1991) (Fig. 6). However, the anterior insula and the prefrontal cortex were the only elements in this processing stream differentially activated during pain intensity discrimination. Other elements such as SI, SII, and the posterior insula do not appear to be differentially activated during intensity discrimination, despite their ability to encode small differences in noxious stimulus intensity (Coghill et al., 1999). However, to minimize effects due to stimulus intensity, analyses of discrimination examined only trials where T1 and T2 stimuli were equally intense. Accordingly, SII and the posterior insular were activated in a statistically indistinguishable manner during both $\mathrm{T} 1$ and $\mathrm{T} 2$ stimuli. taneous suppression of neural activity related to irrelevant stimulus features. The painful contact heat stimuli used in the present investigation as well as the previous investigation both had pronounced intensity and spatial features. The discrimination task required that subjects direct their attention to only one of these sensory features and ignore the other dimensions. Despite the marked presence of both sensory features, attentional modulation appeared to be sufficiently powerful to preferentially amplify intensity processing in the ventral stream and spatial processing in the dorsal stream in a task-dependent manner (Fig. 6). Accordingly, a portion of the activity detected within these preferential processing streams may be associated with top-down signals that are conveyed by bidirectional anatomic connections within each processing stream (Mesulam and Mufson, 1982; Mufson and Mesulam, 1982; Friedman et al., 1986; Felleman and Van Essen, 1991). This bidirectional cortical flow of information may also be crucially important during the delayed match-tosample discrimination task in that it provides a mechanism for comparisons between internal representations of previously experienced stimuli and incoming afferent information (Romo and Salinas, 2003).

During active discrimination of either intensity or spatial features of noxious stimuli, no statistically enhanced activity was detected in either SI or SII. In contrast, the leg representation of SI contralateral to stimulation exhibited significantly decreased activity during intensity discrimination in relation to that evoked by nondiscriminated stimuli (T1). Conversely, SII exhibited significantly decreased activity during spatial discrimination in relation to that evoked by nondiscriminated stimuli (T1) (Oshiro et al., 2007). SI nociceptive neurons have small receptive fields that 
are well suited to provide information about stimulus location (Kenshalo and Isensee, 1983), while SII neurons have larger and more complex receptive fields that can integrate nonspatial information over large portions of the body surface (Fitzgerald et al., 2006). Although highly speculative, this complementary pattern of diminished activity during intensity discrimination and spatial discrimination suggests that SI and SII may play important roles in the selection of the sensory features to be processed (Fig. 6). However, instead of amplifying relevant information, these structures exhibit decreased activity that is consistent with diminished processing of nondiscriminated sensory features. Loss of top-down inputs to either SI and/or SII may result in disruption of spatiotemporal tuning and distorted sensory experiences such as those that occur during central pain.

Both intensity discrimination and spatial discrimination activated portions of the ACC that receive input from both the dorsal and ventral streams. In the case of the dorsal stream, the PPC and DLPFC both project to the ACC (Selemon and Goldman-Rakic, $1985,1988)$. In the case of the ventral stream, the anterior insula is reciprocally connected with the ACC (Vogt and Pandya, 1987). Although both intensity and spatial discrimination tasks activated largely overlapping regions of the right ACC, direct statistical comparisons revealed that portions of the left ACC exhibited a greater magnitude of activation during intensity versus spatial discrimination. This more bilateral pattern of activity is consistent with the bilateral activation of the insula and prefrontal cortex during intensity discrimination. The ACC plays critical roles in aspects of attention, affect, and expectation of pain and may represent an important site in the bidirectional comparison of internally maintained information with external afferent information (Koyama et al., 2005). Since the ACC is activated during both discrimination tasks, this structure appears to be more related to the monitoring of sensory comparisons and the generation of decisions rather than sensory processing per se (Figs. 4, 6) (Bush et al., 2000).

Left hemispheric activation was detected in M1 (hand area) and PMA during both intensity and spatial discrimination. This activation is likely associated with motor planning for pushing the switch to indicate a choice. Both of these structures receive input from the ACC, and together with the ACC, appear to constitute a common apparatus that contributes to the selection and communication of a decision about the nature of the incoming sensory information (Cavina-Pratesi et al., 2006).

Bilateral portions of the head of the caudate, DFC, ACC, and VMPFC were activated during the maintenance of pain intensity representations in short-term memory. Of these areas, the VMPFC has been demonstrated to be clearly involved emotion, negative affect, and episodic memory processing (Bechara et al., 1998; Zald et al., 2002). In contrast, left hemispheric regions such as Broca's area were activated during the memory period during spatial discrimination, a finding consistent with the high frequency of subjects' reports that they used a verbal categorical strategy to remember the stimulus location (Oshiro et al., 2007). However, in the present study, subjects reported that they attempted to remember the intensity of pain itself and only rarely indicated that they used any dimension of the stimulus that may have involved a spatial component (sharpness) (Fig. 5). Accordingly, the activation of the caudate, DFC, ACC, and VMPFC may be related to the maintenance of noncategorical affective feelings in memory in preparation for the future evaluation of incoming afferent nociceptive sensation.

Consistent with other sensory modalities, nonspatial features of painful stimuli are preferentially processed by brain networks that are largely distinct from those that process spatial aspects of pain (Oshiro et al., 2007). Although pain has been viewed as an interoceptive, homeostatic emotion (Craig, 2002), the present data indicate that discrete sensory dimensions of pain are processed in a manner that is clearly analogous to the processing of exteroceptive information of other sensory modalities. The findings that attentional focus can amplify distinctions in the processing of discrete stimulus features underscore how both dorsal and ventral streams are bidirectional pathways and are subject to topdown modulation. However, pain is a complex experience in which multiple dimensions of sensory information are combined to provide a unified conscious event. Thus, information from the dorsal and ventral networks must necessarily be bound together to complete the elaboration of sensory information into a subjective experience of pain.

\section{References}

Anourova I, Nikouline VV, Ilmoniemi RJ, Hotta J, Aronen HJ, Carlson S (2001) Evidence for dissociation of spatial and nonspatial auditory information processing. Neuroimage 14:1268-1277.

Barrett DJ, Hall DA (2006) Response preferences for "what" and "where" in human non-primary auditory cortex. Neuroimage 32:968-977.

Bechara A, Damasio H, Tranel D, Anderson SW (1998) Dissociation Of working memory from decision making within the human prefrontal cortex. J Neurosci 18:428-437.

Bush G, Luu P, Posner MI (2000) Cognitive and emotional influences in anterior cingulate cortex. Trends Cogn Sci 4:215-222.

Cavina-Pratesi C, Valyear KF, Culham JC, Köhler S, Obhi SS, Marzi CA, Goodale MA (2006) Dissociating arbitrary stimulus-response mapping from movement planning during preparatory period: evidence from event-related functional magnetic resonance imaging. J Neurosci 26: 2704-2713.

Coghill RC, Sang CN, Maisog JM, Iadarola MJ (1999) Pain intensity processing within the human brain: a bilateral, distributed mechanism. J Neurophysiol 82:1934-1943.

Cohen MS (1997) Parametric analysis of fMRI data using linear systems methods. Neuroimage 6:93-103.

Colby CL, Goldberg ME (1999) Space and attention in parietal cortex. Annu Rev Neurosci 22:319-349.

Craig AD (2002) How do you feel? Interoception: the sense of the physiological condition of the body. Nat Rev Neurosci 3:655-666.

Darian-Smith I, Johnson KO, LaMotte C, Shigenaga Y, Kenins P, Champness P (1979) Warm fibers innervating palmar and digital skin of the monkey: responses to thermal stimuli. J Neurophysiol 42:1297-1315.

Desimone R, Schein SJ (1987) Visual properties of neurons in area V4 of the macaque: sensitivity to stimulus form. J Neurophysiol 57:835-868.

Felleman DJ, Van Essen DC (1991) Distributed hierarchical processing in the primate cerebral cortex. Cereb Cortex 1:1-47.

Fitzgerald PJ, Lane JW, Thakur PH, Hsiao SS (2006) Receptive field (RF) properties of the macaque second somatosensory cortex: RF size, shape, and somatotopic organization. J Neurosci 26:6485-6495.

Forman SD, Cohen JD, Fitzgerald M, Eddy WF, Mintun MA, Noll DC (1995) Improved assessment of significant activation in functional magnetic resonance imaging (fMRI): use of a cluster-size threshold. Magn Reson Med 33:636-647.

Friedman DP, Murray EA, O’Neill JB, Mishkin M (1986) Cortical connections of the somatosensory fields of the lateral sulcus of macaques: evidence for a corticolimbic pathway for touch. J Comp Neurol 252:323-347.

Friston KJ, Worsley KJ, Frakowiak RJ, Mazziotta JC, Evans AC (1994) Assessing the significance of focal activations using their spatial extent. Hum Brain Mapp 1:214-220.

Friston KJ, Holmes AP, Worsley KJ, Poline JB, Frith CD, Frackowiak RSJ (1995) Statistical parametric maps in functional imaging: a general linear approach. Hum Brain Mapp 2:189-210.

Goodale MA, Milner AD (1992) Separate visual pathways for perception and action. Trends Neurosci 15:20-25.

Josephs O, Turner R, Friston KJ (1997) Event-related fMRI. Hum Brain Mapp 5:243-248. 
Kenshalo DR Jr, Isensee O (1983) Responses of primate SI cortical neurons to noxious stimuli. J Neurophysiol 50:1479-1496.

Koyama T, McHaffie JG, Laurienti PJ, Coghill RC (2005) The subjective experience of pain: where expectations become reality. Proc Natl Acad Sci U S A 102:12950-12955.

Kulkarni B, Bentley DE, Elliott R, Youell P, Watson A, Derbyshire SW, Frackowiak RS, Friston KJ, Jones AK (2005) Attention to pain localization and unpleasantness discriminates the functions of the medial and lateral pain systems. Eur J Neurosci 21:3133-3142.

LaMotte RH, Campbell JN (1978) Comparison of responses of warm and nociceptive C-fiber afferents in monkey with human judgments of thermal pain. J Neurophysiol 41:509-528.

Melzack R, Casey KL (1968) Sensory, motivational, and central control determinants of pain. In: The skin senses (Kenshalo DR, ed), pp 423-435. Springfield, IL: Thomas.

Mesulam MM, Mufson EJ (1982) Insula of the old world monkey. III: Efferent cortical output and comments on function. J Comp Neurol 212:38-52.

Movshon JA, Newsome WT (1996) Visual response properties of striate cortical neurons projecting to area MT in macaque monkeys. J Neurosci 16:7733-7741.

Mufson EJ, Mesulam MM (1982) Insula of the old world monkey. II: Afferent cortical input and comments on the claustrum. J Comp Neurol 212:23-37.

Neal JW, Pearson RCA, Powell TPS (1987) The cortico-cortical connections of area 7b, PF, in the parietal lobe of the monkey. Brain Res 419:341-346.

Oshiro Y, Quevedo AS, McHaffie JG, Kraft RA, Coghill RC (2007) Brain mechanisms supporting spatial discrimination of pain. J Neurosci 27:3388-3394.

Porro CA, Martinig M, Facchin P, Maieron M, Jones AK, Fadiga L (2007) Parietal cortex involvement in the localization of tactile and noxious mechanical stimuli: a transcranial magnetic stimulation study. Behav Brain Res 178:183-189.

Rauschecker JP, Tian B (2000) Mechanisms and streams for processing of "what" and "where" in auditory cortex. Proc Natl Acad Sci U S A 97:11800-11806.

Reed CL, Klatzky RL, Halgren E (2005) What vs. where in touch: an fMRI study. Neuroimage 25:718-726.
Romanski LM, Tian B, Fritz J, Mishkin M, Goldman-Rakic PS, Rauschecker JP (1999) Dual streams of auditory afferents target multiple domains in the primate prefrontal cortex. Nat Neurosci 2:1131-1136.

Romo R, Salinas E (2003) Flutter discrimination: neural codes, perception, memory and decision making. Nat Rev Neurosci 4:203-218.

Sakata H, Kusunoki M (1992) Organization of space perception: neural representation of three-dimensional space in the posterior parietal cortex. Curr Opin Neurobiol 2:170-174.

Selemon LD, Goldman-Rakic PS (1985) Longitudinal topography and interdigitation of corticostriatal projections in the rhesus monkey. J Neurosci 5:776-794.

Selemon LD, Goldman-Rakic PS (1988) Common cortical and subcortical targets of the dorsolateral prefrontal and posterior parietal cortices in the rhesus monkey: evidence for a distributed neural network subserving spatially guided behavior. J Neurosci 8:4049-4068.

Talairach J, Tournoux P (1988) Co-planar stereotaxic atlas of the human brain. New York: Thieme Medical.

Tanaka K (1997) Mechanisms of visual object recognition: monkey and human studies. Curr Opin Neurobiol 7:523-529.

Treede RD, Kenshalo DR, Gracely RH, Jones AK (1999) The cortical representation of pain. Pain 79:105-111.

Ungerleider LG, Haxby JV (1994) 'What' and 'where' in the human brain. Curr Opin Neurobiol 4:157-165.

Vogt BA, Pandya DN (1987) Cingulate cortex of the rhesus monkey: II. Cortical afferents. J Comp Neurol 262:271-289.

Woolrich MW, Ripley BD, Brady M, Smith SM (2001) Temporal autocorrelation in univariate linear modeling of FMRI data. Neuroimage 14: $1370-1386$.

Worsley KJ, Evans AC, Marrett S, Neelin P (1992) A three-dimensional statistical analysis for CBF activation studies in human brain. J Cereb Blood Flow Metab 12:900-918.

Zald DH, Mattson DL, Pardo JV (2002) Brain activity in ventromedial prefrontal cortex correlates with individual differences in negative affect. Proc Natl Acad Sci U S A 99:2450-2454.

Zeki S (1983) Colour coding in the cerebral cortex: the reaction of cells in monkey visual cortex to wavelengths and colours. Neuroscience 9:741765. 